\title{
Stress concentrations at an oblique hole in a thick plate
}

\section{P. Stanley and A. G. Starr \\ University of Manchester}

\section{Summary}

An empirical equation has been obtained for the elastic stress concentration factor at an isolated oblique circular- cylindrical hole in thick flat plate subjected to a uniform, arbitrarily oriented, uniaxial tension. The equation is presented and its development is outlined in this note.

Keywords Stress concentration factor; Empirical equation; Surface fit optimisation; Oblique hole; Thick plate.

\section{Introduction}

An oblique hole in a thick flat plate is shown in Fig.1. The hole is circular-cylindrical and the plate thickness is of the order of the hole diameter or greater; the applied stress is a uniform uniaxial tension in the plane of the plate. The plate edges are assumed to be remote from the hole. The definitive variables are

(i) the angle $\alpha$ between the hole-axis and the plate normal (i.e. the angle of obliquity);

(ii) the ratio of plate thickness (h) to hole diameter (d), h/d;

(iii) the angle $\phi$ between the direction of the applied stress and the major axis of the elliptic intersection of the hole in the surface plane of the plate.

The results of a parametric photoelastic study of the stress distribution at such a hole were presented in ref. 1 for values of the variables $\mathrm{h} / \mathrm{d}=1.33,2.00$ and 3.00 (approx.); $\phi=0,30^{\circ}, 60^{\circ}$ and $90^{\circ}$; and $\alpha=0,15^{\circ}, 30^{\circ}, 45^{\circ}$, and $60^{\circ}$. (For $\mathrm{h} / \mathrm{d}=2.00$, the $\phi=30^{\circ}$ series was not included.) 
Specific stress values were given in non-dimensional form as stress indices i.e. stress divided by the applied stress. The maximum stress index at each hole (i.e. the stress concentration factor), designated $\mathrm{I}_{1 \mathrm{MAX}}$, is tabulated in ref. 1; values are reproduced here in Table 1 for ease of reference. Values for the case of $\mathrm{h} / \mathrm{d} \approx 3.00$ are plotted in Fig.2a to illustrate typical trends.

Details of the positions of the maximum stress indices, $\mathrm{I}_{1 \mathrm{MAX}}$, on the hole surface, are also given in ref. 1. For $\alpha=0$, the maximum index occurs at the mid-plane position at the ends of the diameter normal to the applied load direction. With increasing obliquity, $\mathrm{I}_{1 \mathrm{MAX}}$ moves progressively from the mid-plane position, until for $\alpha=60^{\circ}$ the maximum occurs at the rim of the hole. In general, the position of $\mathrm{I}_{1 \mathrm{MAX}}$ is close to the point on the hole surface where the tangent is parallel to the applied load direction. However, for $\alpha$ values greater than $45^{\circ}$, the position of $\mathrm{I}_{1 \mathrm{MAX}}$ is somewhat closer to the 'acute' end of the major axis of the elliptical intersection (end 'a' in Fig. 1) than the tangent position when $\phi$ is not equal to $90^{\circ}$.

The development of an empirical relationship covering the Table 1 data is the subject of this note.

\subsection{Notation}
$\mathrm{a}, \mathrm{b}$
coefficients
d hole diameter
h plate thickness
$\mathrm{I}_{\text {MAX }} \quad$ maximum experimental stress index
$\mathrm{I}_{\alpha \phi} \quad$ maximum predicted stress index
$\mathrm{I}_{00} \quad$ value of $\mathrm{I}_{\alpha \phi}$ for $\alpha=\phi=0$
$\mathrm{n}$
sample size 

S.D
standard deviation of relative differences $\Delta$ about mean value $\bar{\Delta}$
$\alpha$ angle between hole axis and plate normal (see Fig.1)
relative difference $\left(\mathrm{I}_{\alpha \phi}-\mathrm{I}_{1 \mathrm{MAX}}\right) / \mathrm{I}_{1 \mathrm{MAX}}$
mean value of $\Delta$ within a given data set
$\phi$
angle between direction of applied stress and major axis of the elliptical intersection of the hole in the surface plane of the plate (see Fig.1).

\section{Assumed function}

It was assumed that a satisfactory approximation to the experimental data could be obtained in the form of the quantity $I_{\alpha \phi}$ derived from the following function

$$
I_{\alpha \phi}=I_{00}+\operatorname{acos} \phi(1-\cos \alpha)+b \sin \phi(1-\sec \alpha)
$$

The $\mathrm{I}_{00}$ term is clearly to be identified with the common $\alpha=0$ value of the curves in Fig. $2 \mathrm{a}$; the second term (with negative a) is consistent with the monotonically decreasing curve for $\phi=0$ and the third term (with negative b) with the monotonically increasing curve for $\phi=90^{\circ}$.

Another function, with the $\operatorname{bsin} \phi(1-\sec \alpha)$ term in equation (1) replaced by bsin $\phi \tan \alpha$, was also studied, but in general the equation (1) function proved the more satisfactory.

\section{Determination of $I_{00}$, $a$ and $b$}

Values of the three fitting parameters ( $\mathrm{I}_{00}$, $\mathrm{a}$ and $\mathrm{b}$ ) in equation (1) were determined for which the sum of the squares of the relative differences between the predicted values $\left(I_{\alpha \phi}\right)$ and the corresponding actual values $\left(\mathrm{I}_{1 \mathrm{MAX}}\right)$ of the stress concentration factor in a particular data set $\left(\Sigma\left[\left(I_{\alpha \phi}-I_{1 M A X}\right) / I_{1 M A X}\right]^{2}\right)$ was a minimum. For this purpose the experimental results and computations were tabulated using Microsoft Excel version 7.0 running on a Pentium PC. The 
least squares fits were minimised using a Newton-Raphson search. The running time was negligible. The whole exercise entailed approximately 20 runs.

Two approaches were adopted. The first was to determine least-squares fit values of $\mathrm{I}_{00}$, $\mathrm{a}$ and $\mathrm{b}$ for each of the three separate data sets detailed in Table 1. For the $\mathrm{h} / \mathrm{d} \approx 3.00$ set, as an example, the results were

$$
\begin{aligned}
& \mathrm{I}_{00}=3.404 \\
& \mathrm{a}=-3.047 \\
& \mathrm{~b}=-2.046
\end{aligned}
$$

Values of $\mathrm{I}_{\alpha \phi}$ obtained from equation (1) using these values of $\mathrm{I}_{00}$, a and b are tabulated in Table $2 \mathrm{a}$, and the relative differences $\left[\left(\mathrm{I}_{\alpha \phi^{-}}-\mathrm{I}_{1 \mathrm{MAX}}\right) / \mathrm{I}_{1 \mathrm{MAX}}\right]$ between corresponding predicted and experimental values are given in Table $2 \mathrm{~b}$. It can be seen that these relative differences were within the range $+6.1 \%$ to $-6.0 \%$. The mean value was $-0.1 \%$ and the standard deviation about the mean (S.D.) was $3.6 \%$.

Least squares fit values of $\mathrm{I}_{00}$, $\mathrm{a}$ and $\mathrm{b}$, the relative difference range and the standard deviation of the relative differences are given in Table 3 for each data set.

It has been concluded previously [1] that

"For a given hole obliquity $(\alpha)$ and a given load direction $(\phi)$ the values of the principal stress indices $\left(\mathrm{I}_{1 \mathrm{MAX}} \ldots ..\right)$ are not significantly dependent upon the ratio of plate thickness $(\mathrm{h})$ to hole diameter $(\mathrm{d})$ over the range $1.33<\mathrm{h} / \mathrm{d}<3.0$ " 
With this in mind, as a second approach, least squares fit values of $\mathrm{I}_{00}$, a and $\mathrm{b}$ were obtained for the average $\mathrm{I}_{1 \mathrm{MAX}}$ values for each $\alpha, \phi$ combinations in Table 1 , and then , using these $\mathrm{I}_{00}$, a and $\mathrm{b}$ values, the relative difference distribution, mean and standard deviation for each separate data set were obtained.

Least squares fit values for the average data set were:

$$
\begin{aligned}
& \mathrm{I}_{00}=3.359 \\
& \mathrm{a}=-2.884 \\
& \mathrm{~b}=-2.238
\end{aligned}
$$

Average experimental values, values obtained from equation (1) using the above values of $\mathrm{I}_{00}$, a and $b$, and relative differences are tabulated in Table 4 . The relative difference range was $+6.0 \%$ to $-6.3 \%$; the mean value was $-0.34 \%$, and the standard deviation was $3.7 \%$. The relative difference range, mean and standard deviation for each separate data set, obtained using the same values of $\mathrm{I}_{00}$, $\mathrm{a}$ and $\mathrm{b}$, are given in Table 5. It can be seen that the equation

$$
\mathrm{I}_{\alpha \phi}=3.36-2.88 \cos \phi(1-\cos \alpha)-2.24 \sin \phi(1-\sec \alpha)
$$

provides maximum stress indices which differ from experimental values by no more than $+9.0 \%$ to $-7.8 \%$. The greatest mean relative difference value was $-1.6 \%$ and the standard deviations were approximately $4 \%$.

To facilitate comparison with experimental data, the variations of $\mathrm{I}_{\alpha \phi}$ with $\alpha$ for $\phi=0,30^{\circ}, 60^{\circ}$ and $90^{\circ}$, as derived from equation (2), are shown in Fig. 2 b. 


\section{Discussion}

There are 47 independent data items in Table 1. The distribution of the relative differences between values of $I_{\alpha \phi}$ calculated from equation (2) and corresponding values of $I_{1 M A X}$ is shown in Fig 3 (the plotted 'number' is the number of relative differences greater than the indicated percentage value). The average relative difference is $-0.5 \%$. 36 of the 47 relative differences (77\%) are less than 5\% (numerically) and only $6(13 \%)$ are greater than $7 \%$. The combination of $\alpha$ and $\phi$ at which the extreme difference values tabulated in Table 5 occurred appeared to be randomly distributed; there was no indication of a tendency for the extreme values to occur at a particular co-ordinate combination.

It follows from the form of equation (1) that the gradient of the $I_{\alpha \phi}$ versus $\alpha$ curve is zero at $\alpha=0$ for all $\phi$. It can be shown that for all $\phi$ values less than $\tan ^{-1} \mathrm{a} / \mathrm{b}$, a second zero gradient (i.e. a turning point or minimum) will also occur in the curve. As a consequence, using the $\mathrm{a}$ and $\mathrm{b}$ values in equation (2), such minima are predicted in the $\phi=0$ and $\phi=30^{\circ}$ curves. For $\phi=0$ this minimum occurs at $\alpha=90^{\circ}$, but since no physical significance can be ascribed to such a hole the deduction is meaningless. For $\phi=30^{\circ}$ the minimum is predicted to occur at $\alpha=48^{\circ}$. A minimum in the $\mathrm{I}_{1 \mathrm{MAX}}$ curve for $\phi=30^{\circ}$ can be seen in Fig.2a in the region of $\alpha=45^{\circ}$. (A similar minimum occurred in the $h / d=1.33$ data [1].) The capability of equation (2) to predict this feature of the experimental data is noteworthy. The minimum $\mathrm{I}_{\alpha \phi}$ value for $\phi=30^{\circ}, \alpha=48^{\circ}$ was approximately 6\% greater than the value indicated in Fig. 2a. 


\section{Conclusion}

Within the range of variables $1.3<\mathrm{h} / \mathrm{d}<3.00$ and $\alpha \leq 60^{\circ}$, the equation

$$
\mathrm{I}_{\alpha \phi}=3.36-2.88 \cos \phi(1-\cos \alpha)-2.24 \sin \phi(1-\sec \alpha)
$$

yields estimates of the maximum stress index (i.e. stress concentration factor) at an oblique hole in a flat plate subjected to a uniform uniaxial tension which differ by no more than $9 \%$ from experimental values.

\section{Reference}

1. Stanley, P. and Day, B.V., " Photoelastic investigation of stresses at an oblique hole in a thick flat plate under uniform uniaxial tension”, J. Strain Anal., 1990,25,157-175 
Table 1: Experimental data ( $\left.\mathrm{I}_{1 \mathrm{MAX}}\right)$

\begin{tabular}{ccccccc}
\hline$\phi(\mathrm{deg})$ & $\mathrm{h} / \mathrm{d}$ & \multicolumn{5}{c}{$\alpha(\mathrm{deg})$} \\
\cline { 3 - 7 } & & 0 & 15 & 30 & 45 & 60 \\
\hline 0 & 1.33 & 3.31 & 3.16 & 2.87 & 2.52 & 2.08 \\
& 2.00 & 3.26 & 3.15 & 3.12 & 2.44 & 1.93 \\
& 3.00 & 3.38 & 3.20 & 2.94 & $2.40^{1}$ & $1.99^{2}$ \\
\hline \multirow{2}{*}{30} & 1.33 & - & 3.23 & 2.98 & 2.95 & 3.30 \\
& 2.00 & - & - & - & - & - \\
& 3.00 & - & 3.25 & 3.26 & $2.88^{1}$ & $3.12^{2}$ \\
\hline 60 & 1.33 & - & 3.38 & 3.60 & 3.65 & $4.78^{3}$ \\
& 2.00 & - & 3.56 & 3.55 & 3.71 & 4.75 \\
& 3.00 & - & 3.47 & 3.68 & $3.64^{4}$ & $4.20^{5}$ \\
\hline 90 & 1.33 & - & 3.73 & 3.96 & 4.32 & $5.64^{3}$ \\
& 2.00 & - & 3.73 & 3.88 & 4.37 & 5.61 \\
& 3.00 & - & 3.55 & 3.90 & $4.52^{4}$ & $5.63^{5}$ \\
\hline
\end{tabular}

$\begin{array}{lll}\text { Notes } & 1: & \mathrm{h} / \mathrm{d}=2.80 \\ & 2: & \mathrm{h} / \mathrm{d}=2.59 \\ 3: & \mathrm{h} / \mathrm{d}=1.20 \\ 4: & \mathrm{h} / \mathrm{d}=2.90 \\ 5: & \mathrm{h} / \mathrm{d}=2.57\end{array}$

Table 2a: $\mathrm{I}_{\alpha \phi}$ from equation (1) for $\mathrm{h} / \mathrm{d} \approx 3.00$

\begin{tabular}{cccccc}
\hline$\phi(\operatorname{deg})$ & \multicolumn{5}{c}{$\alpha(\mathrm{deg})$} \\
\cline { 2 - 6 } & 0 & 15 & 30 & 45 & 60 \\
\hline 0 & 3.40 & 3.30 & 3.00 & 2.51 & 1.88 \\
30 & 3.40 & 3.35 & 3.21 & 3.05 & 3.11 \\
60 & 3.40 & 3.41 & 3.47 & 3.69 & 4.41 \\
90 & 3.40 & 3.48 & 3.72 & 4.25 & 5.45 \\
\hline
\end{tabular}

Table 2b: $\left(\mathrm{I}_{\alpha \phi}-\mathrm{I}_{1 \mathrm{MAX}}\right) / \mathrm{I}_{1 \mathrm{MAX}} \times 100$ for $\mathrm{h} / \mathrm{d} \approx 3.00$

\begin{tabular}{cccccc}
\hline$\phi(\operatorname{deg})$ & \multicolumn{5}{c}{$\alpha(\mathrm{deg})$} \\
\cline { 2 - 6 } & 0 & 15 & 30 & 45 & 60 \\
\hline 0 & $0.7 \%$ & $3.1 \%$ & $1.9 \%$ & $4.6 \%$ & $-5.5 \%$ \\
30 & $0.7 \%$ & $3.1 \%$ & $-1.6 \%$ & $6.1 \%$ & $-0.4 \%$ \\
60 & $0.7 \%$ & $-1.6 \%$ & $-5.6 \%$ & $1.4 \%$ & $5.1 \%$ \\
90 & $0.7 \%$ & $-2.1 \%$ & $-4.6 \%$ & $-6.0 \%$ & $-3.2 \%$ \\
\hline
\end{tabular}


Table 3: Least squares fit values of $I_{00}$, $a$ and $b$, relative difference ranges, mean values and standard deviations

\begin{tabular}{|c|c|c|c|c|c|c|}
\hline $\mathrm{h} / \mathrm{d}$ & $\mathrm{I}_{00}$ & $\mathrm{a}$ & $\mathrm{b}$ & Difference range & Mean & S.D. \\
\hline 1.33 & 3.320 & -2.667 & -2.323 & $+7.1 \%-8.8 \%$ & $-0.14 \%$ & $3.88 \%$ \\
\hline 2.00 & 3.361 & -2.888 & -2.374 & $+3.6 \%-7.6 \%$ & $-0.12 \%$ & $3.61 \%$ \\
\hline 3.00 & 3.404 & -3.047 & -2.046 & $+6.1 \%-6.0 \%$ & $-0.12 \%$ & $3.62 \%$ \\
\hline
\end{tabular}

Table 4: $\quad$ a) averaged experimental data

b) calculated $\mathrm{I}_{\alpha \phi}$ data

c) relative differences $(\%)$

(a)

\begin{tabular}{crrrrr}
\hline$\phi(\operatorname{deg})$ & \multicolumn{5}{c}{$\alpha(\mathrm{deg})$} \\
\cline { 2 - 6 } & 0 & 15 & 30 & 45 & 60 \\
\hline 0 & 3.317 & 3.170 & 2.977 & 2.453 & 2.000 \\
30 & 3.317 & 3.240 & 3.120 & 2.915 & 3.210 \\
60 & 3.317 & 3.470 & 3.610 & 3.667 & 4.577 \\
90 & 3.317 & 3.670 & 3.913 & 4.403 & 5.627 \\
\hline
\end{tabular}

(b)

\begin{tabular}{crrrrr}
\hline$\phi(\operatorname{deg})$ & \multicolumn{5}{c}{$\alpha(\mathrm{deg})$} \\
\cline { 2 - 6 } & 0 & 15 & 30 & 45 & 60 \\
\hline 0 & 3.36 & 3.26 & 2.97 & 2.51 & 1.92 \\
30 & 3.36 & 3.31 & 3.20 & 3.09 & 3.23 \\
60 & 3.36 & 3.38 & 3.47 & 3.74 & 4.58 \\
90 & 3.36 & 3.44 & 3.71 & 4.29 & 5.60 \\
\hline
\end{tabular}

(c)

\begin{tabular}{crrrrr}
\hline$\phi(\mathrm{deg})$ & \multicolumn{5}{c}{$\alpha(\mathrm{deg})$} \\
\cline { 2 - 6 } & 0 & 15 & 30 & 45 & 60 \\
\hline 0 & $1.3 \%$ & $2.9 \%$ & $-0.1 \%$ & $2.5 \%$ & $-4.1 \%$ \\
30 & $1.3 \%$ & $2.3 \%$ & $2.5 \%$ & $6.0 \%$ & $0.6 \%$ \\
60 & $1.3 \%$ & $-2.6 \%$ & $-4.0 \%$ & $2.0 \%$ & $0.0 \%$ \\
90 & $1.3 \%$ & $-6.3 \%$ & $-5.3 \%$ & $-2.7 \%$ & $-0.5 \%$ \\
\hline
\end{tabular}

Table 5: Least squares fit values of $I_{00}$, a and $b$, from averaged data, with relative difference ranges, means, and standard deviations

\begin{tabular}{|c|c|c|c|c|c|c|}
\hline $\mathrm{h} / \mathrm{d}$ & $\mathrm{I}_{00}$ & $\mathrm{a}$ & $\mathrm{b}$ & Difference range & Mean & S.D. \\
\hline average & 3.360 & -2.884 & -2.238 & $+6.0 \%-6.3 \%$ & $-0.34 \%$ & $3.37 \%$ \\
\hline 1.33 & " & “ & “ & $+7.3 \%-7.8 \%$ & $-0.51 \%$ & $4.41 \%$ \\
\hline 2.00 & " & " & " & $+3.5 \%-7.8 \%$ & $-1.59 \%$ & $3.56 \%$ \\
\hline 3.00 & " & " & “ & $+9.0 \%-5.8 \%$ & $+0.21 \%$ & $4.36 \%$ \\
\hline
\end{tabular}




\section{Figure captions}

Figure 1: Oblique hole in plate subjected to uniaxial stress

Figure 2a: Experimental data for $\mathrm{h} / \mathrm{d} \approx 3.00$

Figure 2b: Maximum stress indices derived from equation (2).

Figure 3: Number of $\Delta$ values numerically greater than indicated percentage value (Note: The values of 20 and 27 at $0 \%$ are the numbers of positive and negative relative differences, respectively.) 
Figure 1: Oblique hole in plate subjected to uniaxial stress

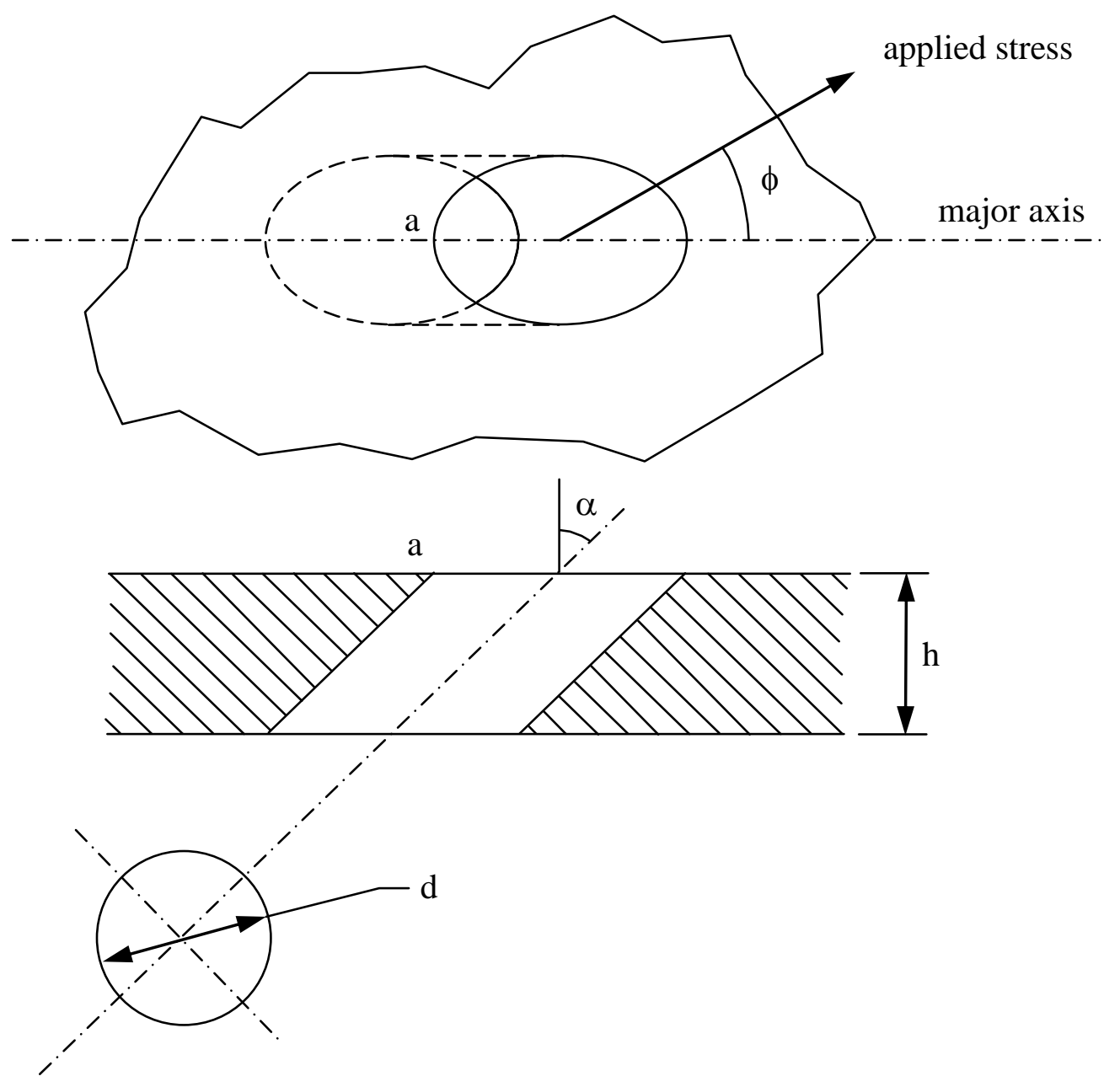


Figure 2a: Experimental data for $\mathrm{h} / \mathrm{d} \approx 3.00$

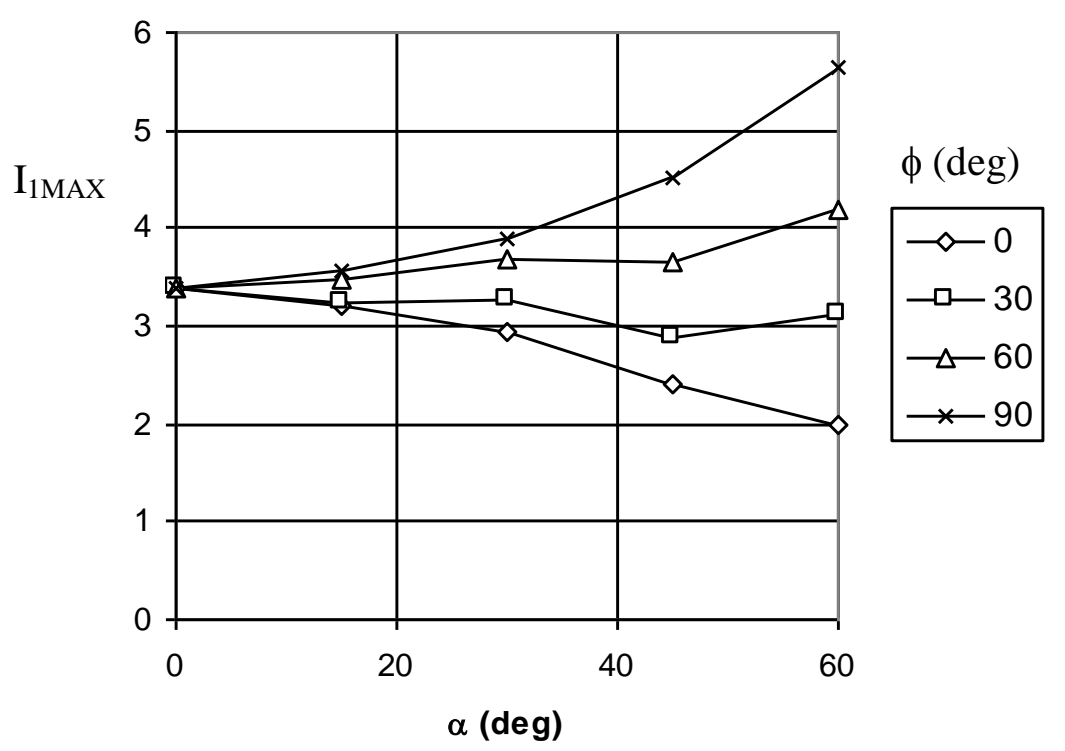


Figure 2b: Maximum stress indices derived from equation (2).

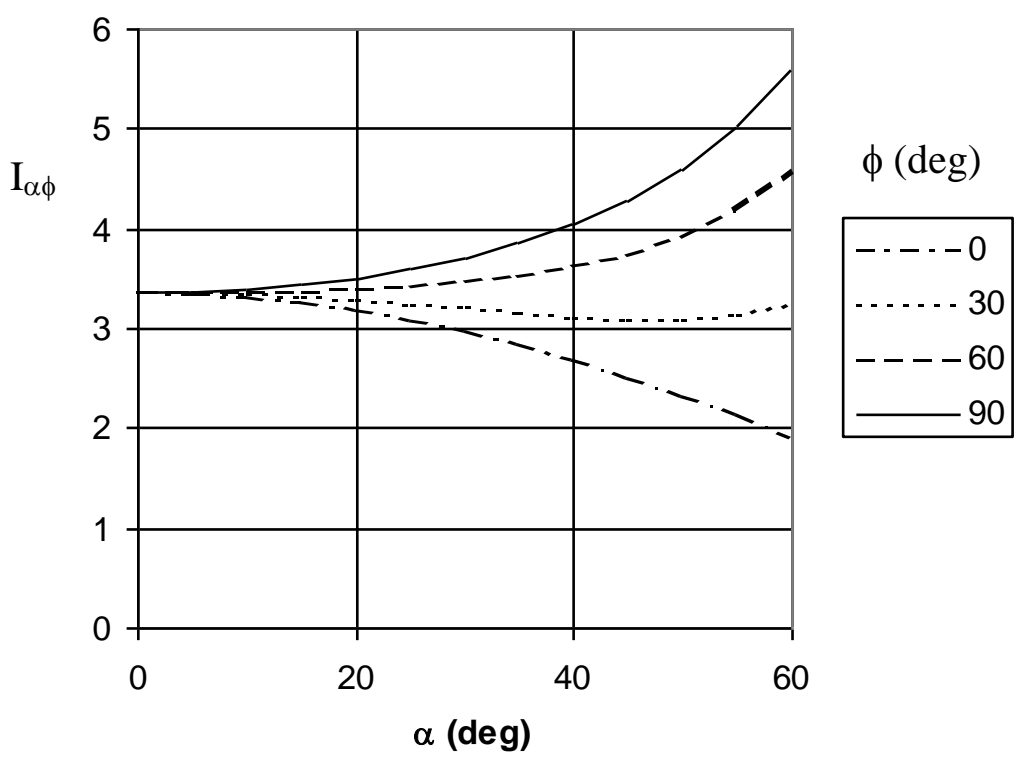


Figure 3: Number of $\Delta$ values numerically greater than indicated percentage value

(Note: The values of 20 and 27 at $0 \%$ are the numbers of positive and negative relative differences, respectively.)

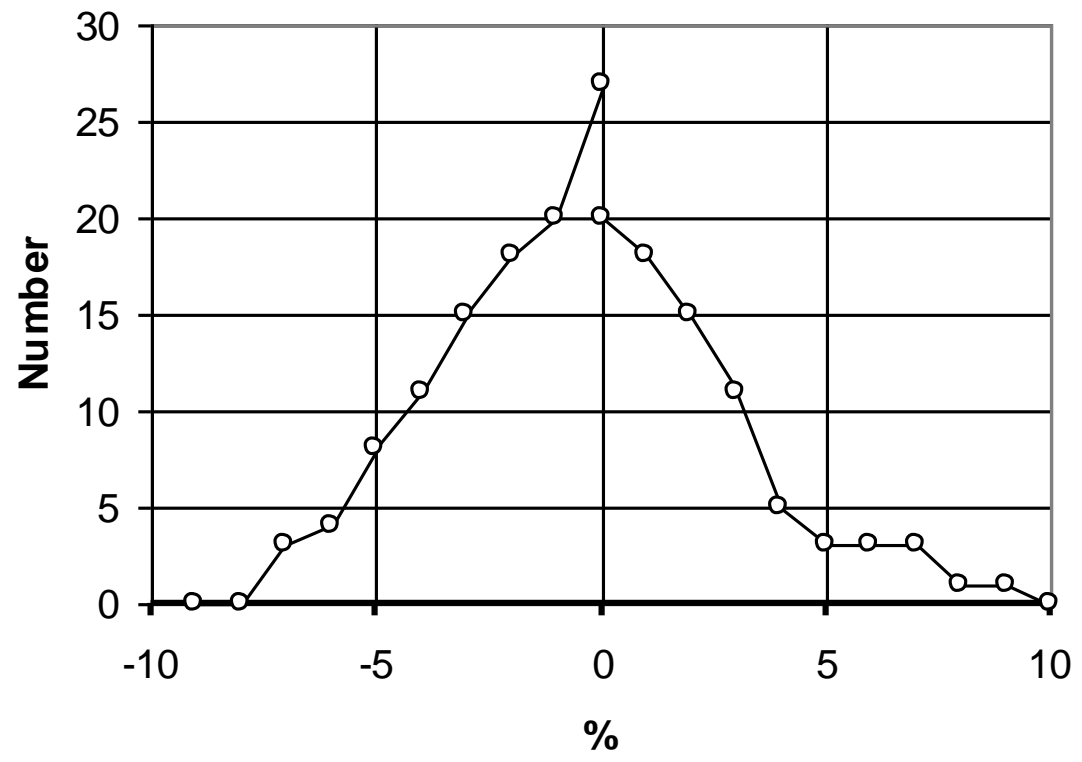

